\title{
Synthesis of a Novel MIP Thin Layer and Monodisperse MIP- Nanoparticles for Detection of Creatinine
}

\author{
Shahin Haghdoust ${ }^{1}$, Peter A. Lieberzeit ${ }^{2 *}$, \\ University of Vienna, Department of Physical Chemistry, Waehringer Strasse 42, \\ A-1090, Vienna, Austria \\ Peter.Lieberzeit@univie.ac.at
}

\begin{abstract}
Herein we report molecularly imprinted polymers (MIP) to design a novel biomimetic assay for the detection of creatinine, which is evaluation bioindicator of renal function and muscle damage. Creatinine MIPs both as thin films and nanoparticles (NPs) were synthesized by precipitation polymerization and characterized with quartz crystal microbalance (QCM) and dynamic light scattering (DLS). Selectivity was verified against creatine. The experimental results show the limit of detection nearly $41 \mathrm{ppm}$ for MIP NPs and $121 \mathrm{ppm}$ for MIP thin film. Selectivity factor recorded four-time higher amount for MIP NPs in comparison with MIP thin film.
\end{abstract}

Key words: QCM, molecularly imprinted polymer, creatinine, nanoparticles.

\section{Introduction}

Creatinine is the end product of creatine catabolism. The measurement of creatinine levels in human blood and urine is clinically essential, because it partially reflects renal, muscular and thyroid functions [1].

Molecularly imprinted polymers (MIPs) are artificially synthesized polymeric materials with specific molecular recognition abilities that have proven highly interesting biomimetic receptors. They are synthesized from monomer and crosslinker around a template molecule. Advantages of MIPs include selectivity, physicochemical, stability, ruggedness and sensitivity combined with comparably low cost [2]. Herein we report synthesis and application of creatinine MIP, both as thin film and nanoparticles for sensing purposes.

\section{Preparing creatinine MIP}

To synthesize creatinine MIP thin films, creatinine was dissolved in Tetrahydrofuran (THF); Methacrylic acid (MAA) and 2vinylpyridine were employed as the monomers, Divinyl-benzene (DVB) as the cross-linker, and AIBN as the initiator. The non-imprinted (NIP) sample was prepared in the same manner without template. After thermal polymerization until reaching the gel-point, the oligomer was spin-coated onto the quartz electrode and hardened overnight. Imprinted polymer NPs are based on the same polymer recipe, as MIP thin films with the exception of switching the solvent from THF to acetonitrile, which leads to precipitation. Then resulting particles were rinsed with distilled water and acetonitrile six times and centrifuged. Collected particles were placed in the oven with $100^{\circ} \mathrm{C}$ for $24 \mathrm{~h}$ to dry. NIP NPs were synthesized in the same way without template for using as a reference. For spin coating, MAA was added to stabilize the MIP NPs on the QCM electrodes.

\section{Results and Discussion}

Polymer recipes published by [3] Miura et al. were modified to prepare MIP thin films on QCM electrodes. For that purpose 2vinylpyridine was selected as the co-monomer in addition to MAA to enhancing the efficiency of polymer chains to mimic creatinine shape with increased precision during the polymerization process.

In a first step, the polymer was optimized by varying the ratio between two monomers in six different volume steps. During screening by QCM measurements, a ratio of 1:1 turned out optimal. The results of sensor characteristic measurements for that material are shown in Figure 2a. It clearly reveals concentration dependent sensor signals of the system. Furthermore, the MIP reaches about five times the frequency shifts of the NIP thus clearly proving feasibility of imprinting. 
Moreover, selectivity of the MIP layer was verified with creatine as shown in Figure. $2 \mathrm{~b}$. Evidently, the MIP prefers its own template over the competing compound by almost half an order of magnitude.

a)

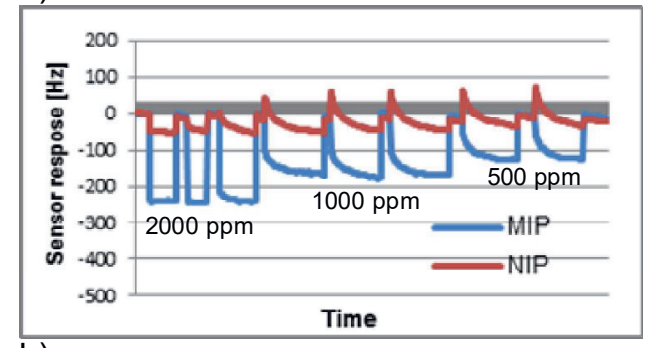

b)

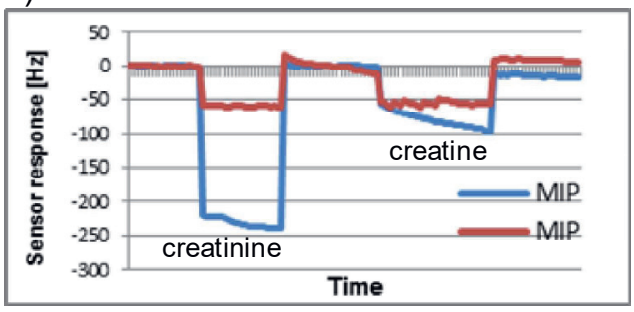

Fig. 2a. sensor response of MIP thin layer for three different analyte concentration; Fig $2 b$. Selectivity test of Creatinine MIP sensor against Creatine.

MIP NPs were assessed according to particles size by dynamic light scattering (DLS) and sensor responses on QCM. Monodispersed creatinine MIP NPs were manufactured with the average size of $304 \mathrm{~nm}$ shown in Figure 3 showing DLS characterization results.

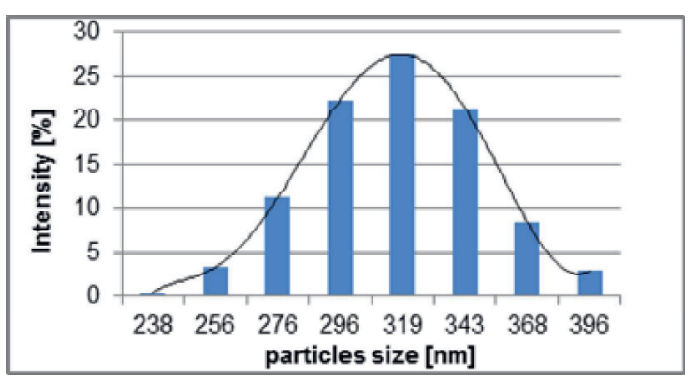

Fig. 3. Particle size distribution measured by DLS.

\section{References}

[1] J. Killard, R. Smyth, Creatinine biosensors: principles and designs. Trends in Biotechnology, 433-437 (2000); doi:10.1016/S01677799(00)01491-8.

[2] H.Munawar. K. Kotova and P. A. Lieberzeit, Sensor, Molecularly Imprinted Polymer Nanoparticles for Formaldehyde Sensing with QCM 1011(2016); doi:10.3390/s16071011.

[3] C.Miura, N. Funaya, H. Matsunaga and J. Haginaka, Monodisperse, molecularly imprinted polymers for creatinine by modified precipitation polymerization and their applications to creatinine assays for human serum and urine, Journal of
Compared to MIP thin films, the corresponding NP layers revealed substantially higher signal in Figure 4a. Evidently, the particle layers give rise to more than three times higher signals on both MIP and NIP. Hence, overall sensitivity is also three times higher. Additionally MIP NPs presented more recognition selectivity of creatinine (Figure 4b.) which may be very interesting to be used of MIP NPs matrix as a reliable sensing strategy for creatinine.

a)
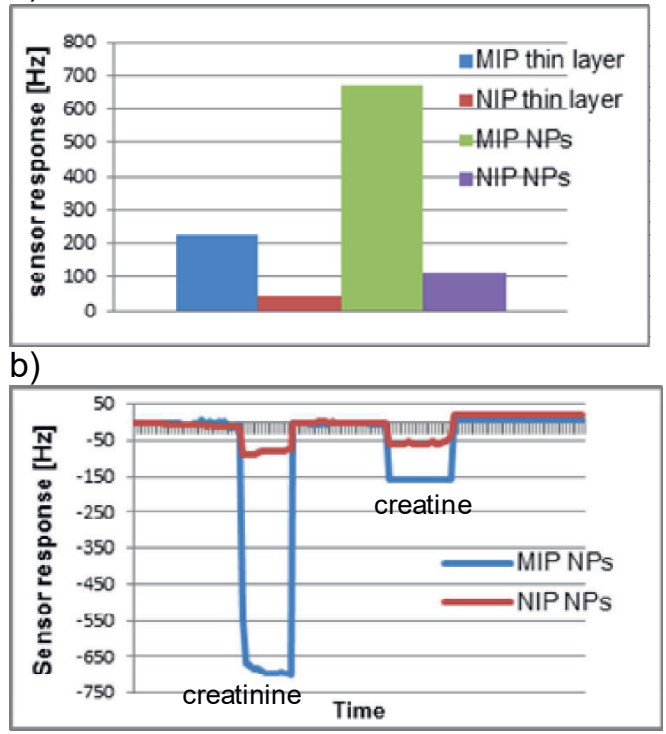

Fig. 4. Compersion the sensor response of MIP and NIP thin-layer sample with MIP NPs and NIP NPs measured with QCM. Fig $2 b$. Selectivity test of Creatinine MIP-NPs sensor against Creatine.

\section{Conclusion}

MIP offer appreciably binding affinity and high selectivity for creatinine on QCM. By switching to NP films this could further be improved by around a factor of three.

Pharmaceutical and Biomedical Analysis, 288294(2013); doi:10.1016/j.jpba.2013.07.038. 